\title{
Shear Modulus of a Coulomb Crystal of Ions: Effects of Ion Motion and Electron Background Polarization
}

\author{
D. A. Baiko* \\ Ioffe Physical-Technical Institute, Politekhnicheskaya 26, 194021 St. Petersburg, Russia \\ St. Petersburg State Polytechnical University, Politekhnicheskaya 29, 195251 St. Petersburg, Russia
}

Received 13 October 2011, revised 10 December 2011, accepted 05 January 2012 Published online 27 February 2012

Key words Coulomb crystal, Yukawa crystal, elastic moduli, shear modulus.

\begin{abstract}
Shear modulus of a body-centered cubic Coulomb crystal of ions is calculated by thermodynamic perturbation theory taking into account ion motion. Classic and quantum regimes of ion motion are considered. The calculations in the classic range of high temperatures agree well with previous Monte Carlo simulations. In this case, the shear modulus is given by a sum of a positive contribution due to the static lattice and a negative $\propto T$ contribution due to the ion motion. In the quantum regime of low temperatures, the contribution to the shear modulus due to the ion motion saturates at a negative constant value, determined by zero-point ion vibrations.

The correction to the static lattice shear modulus due to the polarization of the electron background is also calculated. The background polarization is described by a dielectric function in the linear response formalism. We compare two dielectric functions (simple Thomas-Fermi and more realistic Jancovici) and find dependence of the correction to the shear modulus on the ion charge number and the electron degree of relativity.

The numerical results are approximated by simple analytical formulas. They can be applied to matter in white dwarf cores and neutron star crusts for precise modeling of oscillations of these astrophysical objects.
\end{abstract}

(c) 2012 WILEY-VCH Verlag GmbH \& Co. KGaA, Weinheim

\section{Introduction}

One-component plasma (OCP) is a system of identical point charges (hereafter, ions) with strictly uniform charge-compensating background of opposite charge. The classic OCP is characterized by one parameter $\Gamma=$ $Z^{2} e^{2} /\left(a k_{\mathrm{B}} T\right)$, where $Z$ is the ion charge number, $a=(4 \pi n / 3)^{-1 / 3}$ is the ion sphere radius $(n$ is the ion number density), and $T$ is the temperature. Numerical simulations (e.g., Ref. [1]) show that at $\Gamma \gtrsim 175$ OCP freezes into a body-centered cubic (bcc) Coulomb crystal. Quantum effects become important in OCP at low temperatures $T \ll T_{\mathrm{p}}$, where $T_{\mathrm{p}}=\left(\hbar / k_{\mathrm{B}}\right) \sqrt{4 \pi Z^{2} e^{2} n / M}$ is the ion plasma temperature ( $M$ is the ion mass).

The OCP model provides an excellent description of a number of physical systems as well as is of considerable fundamental interest. The motivation for this work mainly comes from the theory of degenerate stars. It is well known (e.g., Ref. [2]) that matter in the cores of white dwarfs and the crusts of neutron stars can be modeled as a Coulomb crystal of fully pressure-ionized atomic nuclei with nearly incompressible background of strongly degenerate electrons. Eigenmodes of oscillations of these stars have presumably been observed [3] and the accurate knowledge of elastic properties of their matter is now required for qualitative asteroseismology.

Hence, in this paper we analyze the shear modulus of the bcc Coulomb crystal. We discuss the effect of ion vibrations (see also [4]) and present new calculations of the contribution to the shear modulus due to the finite polarizability of the actual electron background.

\section{Deformation and Elastic Moduli}

The Hamiltonian of the Coulomb crystal is a sum of the energy of the static lattice $U_{\mathrm{M}}$ (Madelung energy) and the Hamiltonian of a collection of independent harmonic oscillators $\hat{H}_{2}$ describing ion vibrations (phonon modes): $\hat{H}_{0}=U_{\mathrm{M}}+\hat{H}_{2}$. Kinetic energy of uniform electron background (degenerate electron gas) plays no role.

* Corresponding author. E-mail: baiko@astro.ioffe.ru 
The Madelung energy is constant $U_{\mathrm{M}}=-\zeta N Z^{2} e^{2} / a$, where $\zeta \approx 0.9$ and $N$ is the total number of ions. The Hamiltonian of the phonon gas can be written as $\hat{H}_{2}=\sum_{\mathbf{k} s} \hbar \omega_{\mathbf{k} s}\left(a_{\mathbf{k} s}^{\dagger} a_{\mathbf{k} s}+1 / 2\right)$, where $\mathbf{k}$ and $s$ denote phonon mode wavevector and polarization, respectively, $\omega_{\mathbf{k} s}$ is the phonon frequency, and $a_{\mathbf{k} s}^{\dagger}$ and $a_{\mathbf{k} s}$ are phonon creation and annihilation operators in mode $\mathrm{k} s$.

We apply an infinitely small uniform deformation described by a constant displacement gradient $u_{\alpha \beta}$ to the Coulomb crystal. As a result, the lattice vectors $\mathbf{R}$ will deform $R_{\alpha} \rightarrow R_{\alpha}+u_{\alpha \beta} R_{\beta}$. (Here and below Greek indices denote Cartesian coordinates and summation over repeated indices is assumed.) Accordingly, there will be a variation of the crystal Hamiltonian: $\hat{H}_{0} \rightarrow \hat{H}_{0}+\delta \hat{H}$, where $\delta \hat{H}=\delta U_{\mathrm{M}}+\delta \hat{H}_{2}$.

Variation of the Madelung energy can be expanded in powers of $u_{\alpha \beta}$ as

$$
\frac{\delta U_{\mathrm{M}}}{V}=-P^{\mathrm{st}} u_{\alpha \alpha}+\frac{1}{2} S_{\alpha \beta \gamma \lambda}^{\mathrm{st}} u_{\alpha \beta} u_{\gamma \lambda},
$$

where $V$ is the crystal volume. Pressure of the static lattice $P^{\text {st }}$ and the coefficients $S^{\text {st }}$, which are the elastic moduli of the static Coulomb crystal, were calculated by Fuchs [5].

Variation of the oscillatory part of the crystal Hamiltonian can also be expanded in powers of the displacement gradient and written as $[4,6]$

$$
\delta \hat{H}_{2}=\frac{1}{2} \sum_{\mathbf{k} s s^{\prime}}\left(\Phi_{\alpha \beta}^{\mathbf{k} s s^{\prime}} u_{\alpha \beta}+\frac{1}{2} \Phi_{\alpha \beta \gamma \lambda}^{\mathbf{k} s s^{\prime}} u_{\alpha \beta} u_{\gamma \lambda}\right)\left(a_{\mathbf{k} s}+a_{-\mathbf{k} s}^{\dagger}\right)\left(a_{-\mathbf{k} s^{\prime}}+a_{\mathbf{k} s^{\prime}}^{\dagger}\right),
$$

where expansion coefficients $\Phi$ can be obtained by deforming the crystal dynamic matrix. The expressions for the coefficients $\Phi$ in terms of rapidly converging lattice sums are given in [4].

The Hamiltonian variation $\delta \hat{H}_{2}$ naturally causes variation of the crystal thermodynamic functions. For constant temperature deformations the relevant thermodynamic potential is the free energy $F$. Its variation can be found with the aid of the thermodynamic perturbation theory (e.g., Ref. [7])

$$
\delta F=\sum_{i}\left(\delta \hat{H}_{2}\right)_{i i} w_{i}+\sum_{i, j}^{\prime} \frac{\left|\left(\delta \hat{H}_{2}\right)_{i j}\right|^{2} w_{i}}{E_{i}^{(0)}-E_{j}^{(0)}}+\frac{1}{2 T}\left(\left[\sum_{i}\left(\delta \hat{H}_{2}\right)_{i i} w_{i}\right]^{2}-\sum_{i}\left[\left(\delta \hat{H}_{2}\right)_{i i}\right]^{2} w_{i}\right)+\ldots
$$

In this case, $i$ and $j$ enumerate eigenstates of $\hat{H}_{2}$, i.e., all possible combinations of all non-negative occupation numbers in all phonon modes, while $w_{i}$ and $E_{i}^{(0)}$ are the probability and the unperturbed energy of the $i$-th eigenstate. Expanding Eq. (3) in powers of displacement gradients, one obtains partial pressure associated with ion motion (phonon pressure $P^{\mathrm{ph}}$ ) as well as phonon contributions to the elastic moduli $S_{\alpha \beta \gamma \lambda}^{\mathrm{ph}}$ :

$$
\frac{\delta F}{V}=-P^{\mathrm{ph}} u_{\alpha \alpha}+\frac{1}{2} S_{\alpha \beta \gamma \lambda}^{\mathrm{ph}} u_{\alpha \beta} u_{\gamma \lambda} .
$$

\section{Effective Shear Modulus}

For isotropic material Lagrangian variation of the stress tensor is (e.g., Ref. [8])

$$
\delta \sigma_{\alpha \beta}=K \varepsilon_{\gamma \gamma} \delta_{\alpha \beta}+2 \mu\left(\varepsilon_{\alpha \beta}-\frac{1}{3} \varepsilon_{\gamma \gamma} \delta_{\alpha \beta}\right),
$$

where $K$ and $\mu$ are compression and shear moduli, respectively, while $\varepsilon_{\alpha \beta}=\left(u_{\alpha \beta}+u_{\beta \alpha}\right) / 2$ is the strain tensor. For anisotropic material under non-zero stress in the non-deformed configuration one has (e.g., Ref. [9])

$$
\delta \sigma_{\alpha \beta}=B_{\alpha \beta \gamma \lambda} \varepsilon_{\gamma \lambda}=S_{\alpha \beta \gamma \lambda} \varepsilon_{\gamma \lambda}-P\left(\varepsilon_{\alpha \beta}-\varepsilon_{\gamma \gamma} \delta_{\alpha \beta}\right),
$$

where $P$ is the pressure and $B_{\alpha \beta \gamma \lambda}$ is another set of elastic coefficients. In cubic symmetry there are only three independent $S$ coefficients $S_{1111}, S_{1122}$, and $S_{1212}$. Additionally, $S_{1221}=S_{1212}+P$.

In applications (for instance, calculations of vibration frequencies of white dwarfs or neutron stars) it is usually desirable to express stress in the form of Eq. (5), while, microscopically, the matter is expected to have crystal symmetry. If we rewrite Eq. (5) in the form of Eq. (6), we shall see that $\mu$ must be identified with $S_{1212}$. Alternatively, we can use the isotropisation procedure proposed by Ogata and Ichimaru [10]. Then we obtain effective shear modulus $\mu_{\mathrm{eff}}=\left(S_{1111}-S_{1122}-S_{1221}+4 S_{1212}\right) / 5$. 


\section{Numerical Results}

The total shear modulus of the Coulomb lattice (both $S_{1212}$ and $\mu_{\text {eff }}$ ) is given by a sum of the static lattice and ion motion (i.e. phonon) contributions:

$$
S_{1212}=S_{1212}^{\mathrm{st}}+S_{1212}^{\mathrm{ph}}=0.1827 n Z^{2} e^{2} / a+S_{1212}^{\mathrm{ph}}, \quad \mu_{\mathrm{eff}}=0.1194 n Z^{2} e^{2} / a+\mu_{\mathrm{eff}}^{\mathrm{ph}} .
$$

The static lattice term was evaluated by Fuchs [5]. The total shear modulus of a vibrating classic Coulomb bcc lattice was found by Ogata and Ichimaru [10] by Monte Carlo (MC) simulations. The advantage of the MC method is its ability to take into account anharmonic effects. The disadvantage is extensive computer time requirements and the inability to include quantum effects.

In Ref. [4] we have calculated the phonon contribution to the shear modulus using Eqs. (3) and (4). Evaluation of the sums over $i$ in (3) reduces to a 3D integral over the first Brillouin zone of the nondeformed lattice. This integration can be done numerically by the method proposed in [11]. Our results are shown in Fig. 1 by dashes. At high $T$ the crystal is essentially classic. Calculations in this regime agree well with results of [10] and with our perturbative calculations, in which quantum effects are explicitly excluded (dots in Fig. 1). At low $T \ll T_{\mathrm{p}}$, quantum zero-point vibrations dominate and phonon contributions to elastic moduli approach constant values. In this regime the perturbation theory has a clear advantage over MC method. Our numerical results can be fitted as

$$
\mu_{\mathrm{eff}}^{\mathrm{ph}}=-n k_{\mathrm{B}} T_{\mathrm{p}}\left[0.3686^{3}+136.6\left(T / T_{\mathrm{p}}\right)^{3}\right]^{1 / 3}, \quad S_{1212}^{\mathrm{ph}}=-n k_{\mathrm{B}} T_{\mathrm{p}}\left[0.5903^{3}+439\left(T / T_{\mathrm{p}}\right)^{3}\right]^{1 / 3} .
$$

These fits are displayed in Fig. 1 by thin solid lines. The maximum error of the fits is $2.2 \%$.

The static lattice shear modulus is greater than zero. The phonon contribution is negative and grows with temperature. Naturally, it reduces the lattice resistance to the shear stress.

\section{Polarizable electron background}

Another contribution, $U_{\epsilon}$, to the system energy comes from the polarizability of the electron background. We describe these effect in the linear response formalism by a static $(\omega=0)$ dielectric function $\epsilon(q)$ assuming that all ions are fixed at their lattice nodes $\mathbf{R}$ (thus neglecting modification of the ion motion term by the polarization):

$$
U_{\epsilon}=\frac{Z^{2} e^{2}}{2} \int \frac{\mathrm{d} \mathbf{q}}{(2 \pi)^{3}} \frac{4 \pi}{q^{2}}\left[\frac{1}{\epsilon(q)}-1\right]\left(\sum_{\mathbf{R}} e^{i \mathbf{q} \mathbf{R}}-n \int \mathrm{d} \mathbf{r} e^{i \mathbf{q r}}\right)
$$

(e.g. Ref. [12]). We compare two expressions for the dielectric function. First is the Thomas-Fermi (TF) dielectric function $\epsilon_{\mathrm{TF}}(q)=1+\kappa_{\mathrm{TF}}^{2} / q^{2}$. It describes exponential screening of the Coulomb potential with the screening length equal to $1 / \kappa_{\mathrm{TF}}=\sqrt{\pi \beta / \alpha} /\left(2 k_{\mathrm{F}}\right)$, where $\beta=v_{\mathrm{F}} / c, v_{\mathrm{F}}$ and $k_{\mathrm{F}}$ are Fermi velocity and wavevector of electrons, $\alpha$ is the fine-structure constant, and $c$ is the speed of light. Second expression is the more realistic static dielectric function of degenerate relativistic electron gas at $T=0$ obtained by Jancovici (J) [13].

Polarization contributions to the elastic moduli are obtained by expansion of Eq. (9) (with deformed lattice vectors) in powers of displacement gradients. The calculation with the Jancovici dielectric function $\epsilon_{\mathrm{J}}$ can be performed by switching to a sum over reciprocal lattice vectors $\mathbf{G}$ with the aid of the identity $\sum_{\mathbf{R}} \exp (i \mathbf{q R})=$ $(2 \pi)^{3} n \sum_{\mathbf{G}} \delta(\mathbf{q}-\mathbf{G})$. This sum will converge fairly rapidly because $\left(1 / \epsilon_{\mathrm{J}}\right)-1 \sim q^{-4}$ at large $q$. By contrast, $\left(1 / \epsilon_{\mathrm{TF}}\right)-1 \sim q^{-2}$ at large $q$ and the respective reciprocal lattice sum would converge rather slowly. Therefore, for calculations with $\epsilon_{\mathrm{TF}}$ it is convenient to apply Ewald transformations. Practical formulas for calculations of the polarization contribution to the elastic moduli will be presented elsewhere.

The polarization contribution to the effective shear modulus is also negative. It is shown in Fig. 2 as a function of $Z$ for two values of the electron relativity parameter $x=\hbar k_{\mathrm{F}} / m c$, where $m$ is the electron mass. Dotted and solid lines show calculations with $\epsilon_{\mathrm{J}}$ for $x=1$ and 10 , respectively (filled squares and circles indicate integer $Z$ ). Dash-dotted and dashed curves represent respective results with $\epsilon_{\mathrm{TF}}$. Jancovici dielectric function has a slope change of its $q$-dependence at $q=2 k_{\mathrm{F}}$. This gives rise to a singular structure of the shear modulus seen in Fig. 2. The singularities occur for such $Z$ where lengths of some reciprocal lattice vectors coincide with $2 k_{\mathrm{F}}$. It must be emphasized that $\epsilon_{\mathrm{J}}$ takes into account neither finite temperature of the electron gas nor band structure of electrons in the Coulomb crystal. Both effects are expected to be crucial at and near the singularities. TF results are smooth and on average overestimate slightly the polarization contribution to the shear modulus. 


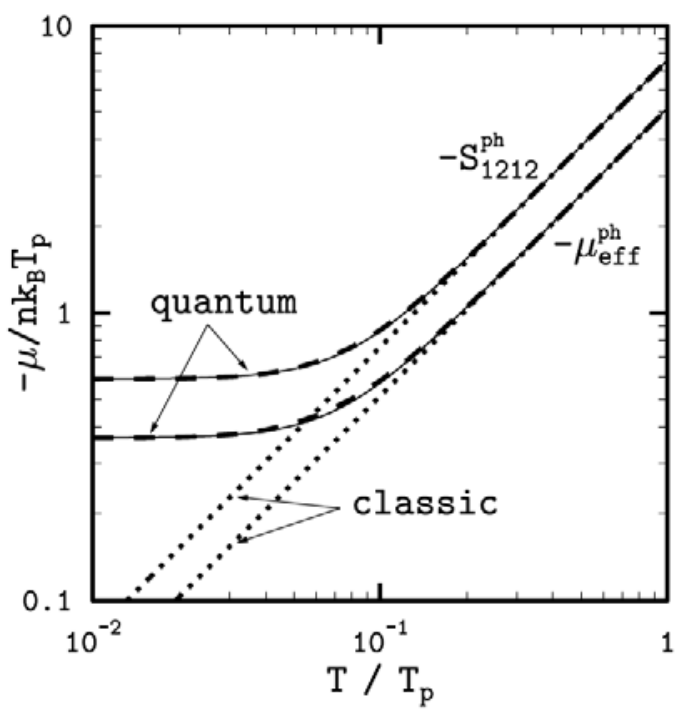

Fig. 1 Phonon elastic coefficient $-S_{1212}^{\mathrm{ph}}$ and effective shear modulus $-\mu_{\mathrm{eff}}^{\mathrm{ph}}$ (upper and lower dashed curves) in units of $n k_{\mathrm{B}} T_{\mathrm{p}}$ vs. $T / T_{\mathrm{p}}$. Solid curves and dots show analytic fits (8) and classic numerical results, respectively.

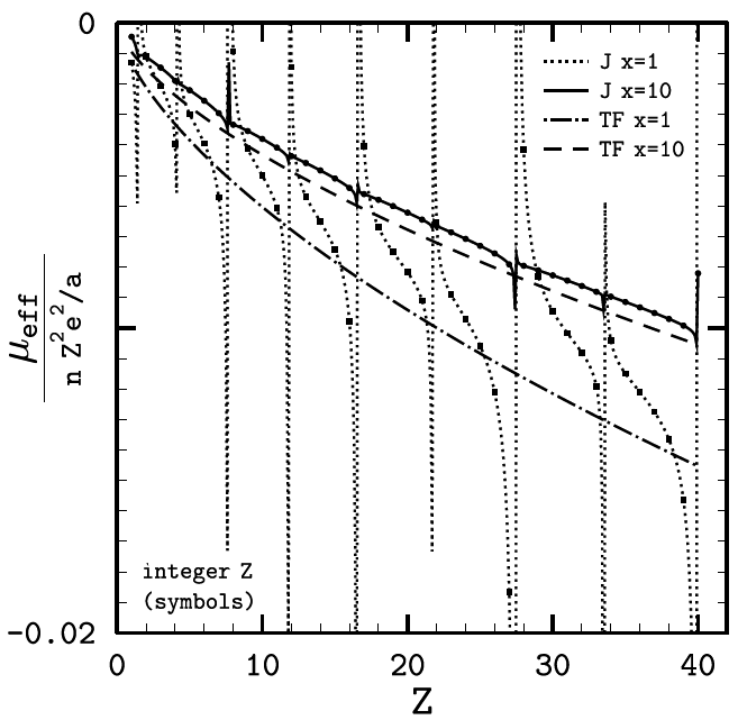

Fig. 2 Correction to the effective shear modulus due to electron background polarization vs. $Z$. Dotted, solid, dashdotted, and dashed lines show results of calculations with $\epsilon_{\mathrm{J}}, x=1,10$, and with $\epsilon_{\mathrm{TF}}, x=1,10$, respectively.

\section{Conclusion}

Effective shear modulus of the bcc Coulomb crystal of ions is calculated taking into account ion motion about their lattice nodes as well as weak polarizability of the charge-compensating electron background. The ion motion contribution to the shear modulus is negative and is determined by a single parameter $T / T_{\mathrm{p}}$ (Fig. 1). Our results are in good agreement with MC simulations at classic (high) temperatures. In quantum temperature range this contribution saturates at a negative constant value, determined by zero-point ion vibrations. The polarization contribution to the shear modulus is also negative and depends on electron degree of relativity and ion charge number (Fig. 2). Two different dielectric functions, Thomas-Fermi and Jancovici, yield similar (on average) results for the polarization correction, with Jancovici dielectric function producing singularities in the $Z$-dependence of the shear modulus. Presumably, these singularities are smoothed out if temperature and bandgap effects are taken into account in the dielectric function. Our results can be applied to matter in white dwarf cores and neutron star crusts for precise modeling of oscillations of these astrophysical objects.

Acknowledgements The work was supported by Ministry of Education and Science of Russian Federation (contract No. 11.G34.31.0001), by RFBR (grant 11-02-00253-a) and by Rosnauka (grant NSh 3769.2010.2).

\section{References}

[1] G.S. Stringfellow, H.E. DeWitt, W.L. Slattery, Phys. Rev. A 41, 1105 (1990).

[2] P. Haensel, A.Y. Potekhin, D.G. Yakovlev, Neutron Stars 1: Equation of State and Structure. Springer, New York 2007.

[3] G.L. Israel et al., ApJ 628, L53 (2005).

[4] D.A. Baiko, MNRAS 416, 22 (2011).

[5] K. Fuchs, Proc. Roy. Soc. London 153, 622 (1936).

[6] M. Born, K. Huang, Dynamical Theory of Crystal Lattices; Izdatelstvo inostrannoi literatury, Moscow 1954.

[7] L.D. Landau, E.M. Lifshitz, Statistical Physics. Part I. Pergamon Press, Oxford 1980.

[8] L.D. Landau, E.M. Lifshitz, Theory of Elasticity. Pergamon Press, Oxford 1986.

[9] D.C. Wallace, Phys. Rev. 162, 776 (1967).

[10] S. Ogata, S. Ichimaru, Phys. Rev. A 42, 4867 (1990).

[11] R.C. Albers, J.E. Gubernatis, Los Alamos Scientific Laboratory Report No. LA-8674-MS 1981.

[12] D.A. Baiko, Phys. Rev. E 66, 056405 (2002).

[13] B. Jancovici, Nuovo Cimento 25, 428 (1962). 